\title{
A Robust Optical Sensor for Remote Multi-Species Detection Combining Frequency-Division Multiplexing and Normalized Wavelength Modulation Spectroscopy
}

\author{
Wenling Jin ${ }^{1,2}$, Hui Zhang ${ }^{1,2}$, Mai Hu ${ }^{1}$, Mengpeng $\mathrm{Hu}^{1,2}{ }^{1}$, Yubin Wei ${ }^{3}$, Jingqiu Liang ${ }^{1}$, Ruifeng Kan ${ }^{1}$ and \\ Qiang Wang ${ }^{1,2, *}$ \\ 1 State Key Laboratory of Applied Optics, Changchun Institute of Optics, Fine Mechanics and Physics, \\ Chinese Academy of Sciences, Changchun 130033, China; jinwenling17@mails.ucas.ac.cn (W.J.); \\ zhanghui195@mails.ucas.ac.cn (H.Z.); humai@ciomp.ac.cn (M.H.); humengpeng19@mails.ucas.ac.cn (M.H.); \\ liangjq@ciomp.ac.cn (J.L.); rfkan@ciomp.ac.cn (R.K.) \\ 2 University of Chinese Academy of Sciences, Beijing 100049, China \\ 3 Institute of Laser, Shandong Academy of Sciences, Jinan 250014, China; wyb9806@qlu.edu.cn \\ * Correspondence: wangqiang@ciomp.ac.cn; Tel.: +86-0431-86176199
}

check for updates

Citation: Jin, W.; Zhang, H.; Hu, M.; $\mathrm{Hu}$, M.; Wei, Y.; Liang, J.; Kan, R.; Wang, Q. A Robust Optical Sensor for Remote Multi-Species Detection Combining Frequency-Division Multiplexing and Normalized Wavelength Modulation Spectroscopy. Sensors 2021, 21, 1073. https:// doi.org/10.3390/s21041073

Academic Editor: Anna Chiara De Luca

Received: 18 December 2020

Accepted: 1 February 2021

Published: 4 February 2021

Publisher's Note: MDPI stays neutral with regard to jurisdictional claims in published maps and institutional affiliations.

Copyright: (c) 2021 by the authors. Licensee MDPI, Basel, Switzerland. This article is an open access article distributed under the terms and conditions of the Creative Commons Attribution (CC BY) license (https:// creativecommons.org/licenses/by/ $4.0 /)$.

\begin{abstract}
By combining frequency-division multiplexing and normalized wavelength modulation spectroscopy, a robust remote multi-species sensor was developed and demonstrated for practical hydrocarbon monitoring. Independently modulated laser beams are combined to simultaneously interrogate different gas samples using an open-ended centimeter-size multipass cell. Gas species of interest are demodulated with the second harmonics to enhance sensitivity, and high immunity to laser power variation is achieved by normalizing to the corresponding first harmonics. Performance of the optical sensor was experimentally evaluated using methane $\left(\mathrm{CH}_{4}\right)$ and acetylene $\left(\mathrm{C}_{2} \mathrm{H}_{2}\right)$ samples, which were separated by a 3-km fiber cable from the laser source. Sub-ppm sensitivity with 1-s time resolution was achieved for both gas species. Moreover, even with large laser intensity fluctuations ranging from 0 to $6 \mathrm{~dB}$, the noise can be kept within 1.38 times as much as that of a stable intensity case. The reported spectroscopic technique would provide a promising optical sensor for remote monitoring of multi hazardous gases with high robustness.
\end{abstract}

Keywords: multi-species sensor; normalized wavelength modulation spectroscopy; frequencydivision multiplexing; remote sensing

\section{Introduction}

Gas monitoring is crucial for safe operation at chemical plants, coal mines, and gas stations, where leaked toxic, flammable, or explosive gases may cause serious accidents [1,2]. Besides, the ability of simultaneous multiple components detection is attracting rising attention in human breath diagnosis [3]. Real-time identification and quantification of different gases of interest are challenging to bridge the potential danger and an early warning. Among numerous metrologies with electrochemistry [4], Fourier Transform Spectrometer [5], etc., laser absorption spectroscopy has proven to be a promising gas analysis method for high selectivity, high sensitivity, and remote sensing [6,7]. Various typical multi-species sensing techniques were afterwards developed based on photoacoustic spectroscopy (PAS), dual-frequency comb (DFC), and laser heterodyne radiometer (LHR) [8-11].

To perform multi-species detection, two requirements should be satisfied. Firstly, gas molecules with distinguishable fingerprint spectra can be effectively illuminated by the light source. Secondly, different absorption features can be separately demodulated without any cross interference. Over past decades, multi-species detection based on laser absorption spectroscopy has been well developed. Given that transitions of different gas species may lay beyond the spectral range of a single laser, an approach with a light source comprising multiple lasers is commonly used. Wu et al. reported a dual-gas 
quartz enhanced photoacoustic spectroscopy (QEPAS) sensor for $\mathrm{H}_{2} \mathrm{O}$ and $\mathrm{C}_{2} \mathrm{H}_{2}$ with two near-infrared lasers, and the excited acoustic signals were separated by two different demodulation frequencies, i.e., the fundamental and the first overtone vibrations of the same QTF [8]. Similarly, Liu et al. employed three diode lasers to demonstrate another $\mathrm{H}_{2} \mathrm{O}, \mathrm{CH}_{4}$, and $\mathrm{CO}_{2}$ sensor, whose acoustic signals were generated with three resonators and separated at different frequencies [9]. With the development of a supercontinuum light source and diode laser with an external cavity (ECDL), even a single laser is currently capable of covering the selected absorption lines of the target gas species. Rieker et al. reported remote multi-species sensing with DFC, covering a spectral range from 5990 to $6260 \mathrm{~cm}^{-1}$, and demonstrated simultaneous measurement of $\mathrm{CO}_{2}, \mathrm{CH}_{4}, \mathrm{H}_{2} \mathrm{O}, \mathrm{HDO}$, and ${ }^{13} \mathrm{CO}_{2}$ over a $2 \mathrm{~km}$ atmospheric path [10]. Differently, Wang et al. utilized the sunlight as the light source to interrogate the earth's atmosphere, of which the $\mathrm{CO}_{2}$ and $\mathrm{CH}_{4}$ columnaveraged abundances were simultaneously acquired by two local oscillators using LHR [11]. However, among most of the above typical techniques, the sensors' performance, especially non-linear spectroscopy, e.g., PAS-based sensors, replies critically on the stability of light power. A stable source power is needed in the gas sensing implementation, otherwise false measurements in practical implementation would appear.

Many efforts are contributed in the optical sensing society to overcome the issues. Constant laser output power over a laser-frequency scan has been achieved by an active servo loop with an acousto-optic modulator (AOM) [12] or an electro-optic modulator (EOM) [13]. This offers an advantage of simplifying enormously the spectral analysis. Besides, influence of laser fluctuation on the spectrum recovery can also be effectively suppressed by a dual-beam regime with balanced detection [14] or a division process [15]. However, for both single-beam and dual-beam regimes, it is still challenging to stabilize the light power coupled on photodetectors, especially for remote sensing after long-distance transmission with inevitable and irregular scattering loss, inconstant transmission loss, even devices' unstable operation. On the contrary, a calibration-free wavelength modulation spectroscopy was proposed for recovering the absorption profile from harsh environments $[16,17]$, by which the unstable laser influences in single-beam regimes could be post processed with careful laser modulation characterization.

In this work, we demonstrated a robust sensor with high immunity to lasers' power noise. Frequency-division multiplexing (FDM) and normalized wavelength modulation spectroscopy are harnessed towards real-time remote multi-species sensing with simpler analysis. Different gas species of interest are simultaneously interacted by the same openended probe with individually modulated fiber-coupler diode lasers, which are combined before interaction and the subsequent coupling on one single photodetector. The target gases are demodulated based on FDM with separate digital lock-in amplifiers (LIA) on a LabVIEW platform. The robustness is enhanced by normalizing to the corresponding first harmonic signals, deriving from the same time-domain signal, to remove the influence of irregular laser intensity fluctuation. With simultaneous $\mathrm{C}_{2} \mathrm{H}_{2}$ and $\mathrm{CH}_{4}$ measurements at a harsh operation condition, the sensor's characterization and performance were experimentally investigated. Besides, only the non-electrified probe serves in the work area while all the others could operate in a much gentler monitoring area, guaranteeing intrinsic safety and stable operation for hazardous gas sensing.

\section{Principle}

The transmission coefficient $\tau(v)$ of laser radiation through a medium length of $L$ is governed by Beer-Lambert law, which relates the transmitted intensity $I_{t}$ to the incident intensity $I_{0}$ as [18]

$$
\tau(v)=\left(\frac{I_{t}}{I_{0}}\right)=\exp [-\alpha(v)] \approx 1-P \chi_{i} L \sum_{j} S_{j}(T) \varphi_{j}(v)
$$

where $\alpha(v)$ represents the spectral absorbance at optical frequency $v, P$ is the total gas pressure, $\chi_{i}$ is the mole fraction of the target specie, $S_{j}(T)$ and $\varphi_{j}(v)$ are the line strength 
and line-shape function of $j$ th absorption feature respectively. The summation accounts for the overlap of adjacent absorption features, which is variable by collisional broadening at different pressures. With wavelength modulation at a frequency of $\omega=2 \pi f$ via the laser injection current, the laser intensity $I_{0}$ is simultaneously modulated but with a WM/IM phase shift and an instantaneous nonlinear response $[19,20]$.

$$
\begin{gathered}
v(t)=\bar{v}+a \cos (\omega t) \\
I_{0}(t)=\bar{I}_{0}[1+\underbrace{i_{0} \cos \left(\omega t+\psi_{1}\right)}_{\text {linear part }}+\underbrace{i_{2} \cos \left(2 \omega t+\psi_{2}\right)}_{\text {nonlinear part }}]
\end{gathered}
$$

where $\bar{v}$ is the center frequency, $a$ is the modulation depth, $\bar{I}_{0}$ is the average laser intensity at $\bar{v}, i_{0}$ and $\psi_{1}$ are the intensity modulation amplitude and phase difference of the linear part, $i_{2}$ and $\psi_{2}$ is the intensity modulation amplitude and phase difference of the nonlinear part. While the spectral absorption can be expanded in a Fourier cosine series as

$$
\tau[\bar{v}+a \cos (\omega t)]=\sum_{k=0}^{k=+\infty} H_{k}(\bar{v}, a) \cos (k \omega t)
$$

where the functions $H_{k}(\bar{v}$, a) are given by

$$
\begin{gathered}
H_{0}(\bar{v}, a)=\frac{1}{2 \pi} \int_{-\pi}^{+\pi}\left[1-P \chi_{i} L \sum_{j} S_{j}(T) \varphi_{j}(\bar{v}+a \cos u)\right] d u \\
H_{k}(\bar{v}, a)=\frac{1}{\pi} \int_{-\pi}^{+\pi}\left[1-P \chi_{i} L \sum_{j} S_{j}(T) \varphi_{j}(\bar{v}+a \cos u)\right] \cos k u d u, \quad k>0
\end{gathered}
$$

The second harmonic component at $2 f$, extracted from the original detector signal with an LIA, is commonly used to perform absorption-based gas sensing. Combining Equations (1)-(6), the absolute magnitude of the $2 f$ signal is given by

$$
\begin{aligned}
& R_{2 f}=\sqrt{\left(X_{2 f}\right)^{2}+\left(Y_{2 f}\right)^{2}}
\end{aligned}
$$

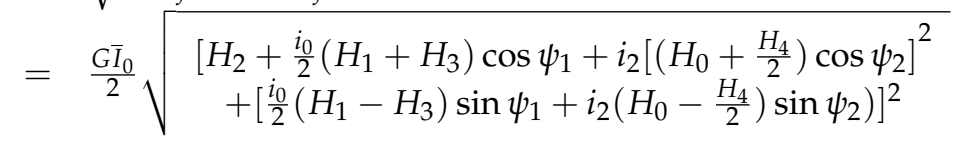

where $G$ is the equivalent gain factor of the detection system, $X_{2 f}$ and $Y_{2 f}$ are the orthogonal components of the second harmonic signal respectively. The demodulated $2 f$ signal is proportional not only to the product of gain factor and the absorption part, which is expressed by the square root function in Equation (7), but also to the laser intensity coupled on the photodetector. Therefore, the stability of $\bar{I}_{0}$ is important to the sensor performance, resulting in a potential risk of destroying the reliability of most absorption-based monitoring systems without reasonable compensation algorithms or stability strategies [8-11,21-23]. The issue could be more critical to scenarios where the laser intensity is very sensitive to the practical environment after a long-path transmission via free-space optics [24] or conductive fiber [25].

Similar to Equation (7), the absolute magnitude of the first harmonic signal, extracted from the same time-domain detector signal, can be expressed by

$$
\begin{aligned}
& R_{1 f}=\sqrt{\left(X_{1 f}\right)^{2}+\left(Y_{1 f}\right)^{2}}
\end{aligned}
$$

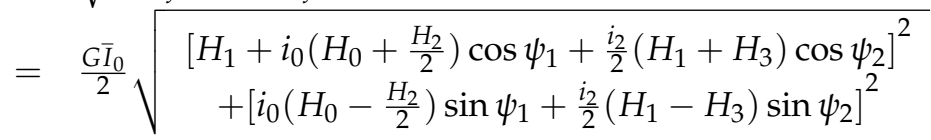


where $X_{1 f}$ and $Y_{1 f}$ are the orthogonal components of the first harmonic signal respectively. In the architecture of wavelength modulation spectroscopy, $1 f$ detection is not preferred for gas demodulation due to distorted waveform with a strong background deriving from residual amplitude modulation (RAM) [6,7]. As analyzed in Equation (8), $1 f$ signal is also proportional to the laser intensity $\bar{I}_{0}$, because of which $R_{1 f}$ would be a candidate to improve the robustness of absorption-based sensors by counteracting both instantaneous and long-term laser intensity variations coupled on the photodetector. The process is described as normalization using different harmonics from the same photodetector signal, which is shown as

$$
\begin{aligned}
& S=R_{2 f} / R_{1 f}= \\
& \sqrt{\frac{\left[H_{2}+\frac{i_{0}}{2}\left(H_{1}+H_{3}\right) \cos \psi_{1}+i_{2}\left[\left(H_{0}+\frac{H_{4}}{2}\right) \cos \psi_{2}\right]^{2}+\left[\frac{i_{0}}{2}\left(H_{1}-H_{3}\right) \sin \psi_{1}+i_{2}\left(H_{0}-\frac{H_{4}}{2}\right) \sin \psi_{2}\right)\right]^{2}}{\left.\left[H_{0}+\frac{H_{2}}{2}\right) \cos \psi_{1}+\frac{i_{2}}{2}\left(H_{1}+H_{3}\right) \cos \psi_{2}\right]^{2}+\left[i_{0}\left(H_{0}-\frac{H_{2}}{2}\right) \sin \psi_{1}+\frac{i_{2}}{2}\left(H_{1}-H_{3}\right) \sin \psi_{2}\right]^{2}}}
\end{aligned}
$$

It can be seen that the normalized wavelength modulation signal is independent of the laser intensity coupled on the photodetector. This characteristic makes it a promising approach to a promoted absorption-based gas sensor immune to unexpected intensity variation before/during/after the interaction.

To extend the technique to a practical versatile gas sensor, capable of simultaneous multi-species sensing, several lasers with different modulation frequencies are combined as one beam to probe the gas molecules at the target area. As shown in Figure 1, the combined light is absorbed by different gas molecules. Harmonics (both $f$ and $2 f$ ) of the species can be distinctly separated in the frequency domain, and be simultaneously picked up by LabVIEW-based digital LIAs. Multi-species concentrations are subsequently demodulated at different demodulation frequencies with a normalizing process.

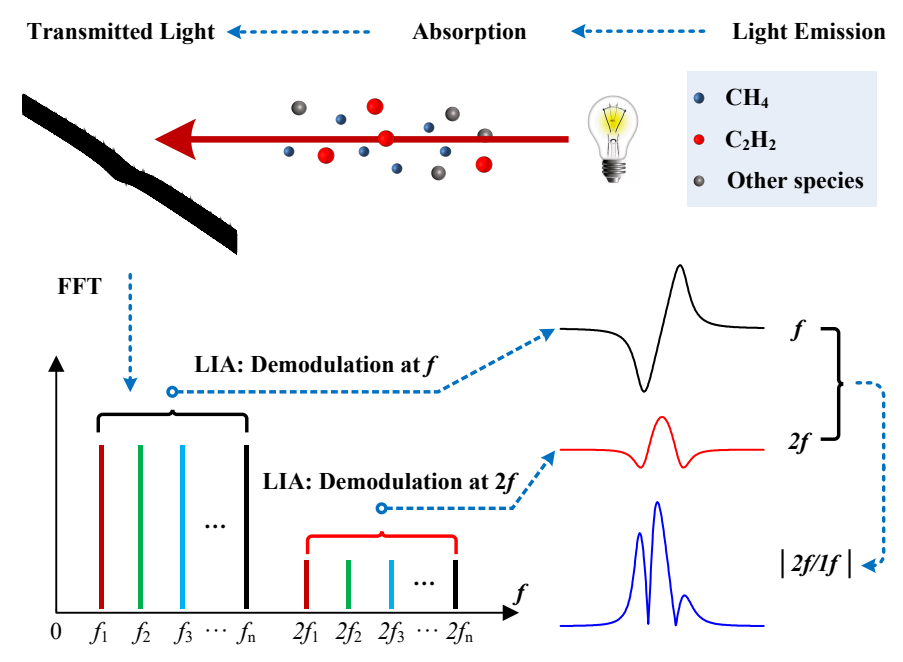

Figure 1. Schematic of the multi-species sensor combining normalized wavelength modulation spectroscopy and frequency-division multiplexing. $f_{1}, f_{2} \ldots f_{\mathrm{n}}$ equal the possible different modulation frequencies, and $2 f_{1}, 2 f_{2} \ldots 2 f_{\mathrm{n}}$ represent the corresponding second harmonic positions in frequency domain. FFT: Fast Fourier Transform; LIA: Lock-in Amplifier.

\section{Instrumentation}

\subsection{Absorption Transition Selection}

Due to the advantages of low transmission loss, intrinsic safety, and a wide operating wavelength range in the near-infrared region, a single-mode fiber (SMF-28 $\left.\mathrm{e}^{+}, \mathrm{Corning}\right)$ is employed to guide the lasers for remote multi-species interrogation. As important hydrocarbons in industry and environment [26,27], methane $\left(\mathrm{CH}_{4}\right)$ and acetylene $\left(\mathrm{C}_{2} \mathrm{H}_{2}\right)$ are selected to demonstrate the implementation of multi-species sensing. 
Figure 2 depicts the calculated absorption spectra of $\mathrm{CH}_{4}$ in $6043-6049 \mathrm{~cm}^{-1}$ and $\mathrm{C}_{2} \mathrm{H}_{2}$ in 6531.5-6538.5 $\mathrm{cm}^{-1}$ based on the HITRAN database [28], showing typical transitions of $\mathrm{CH}_{4}$ and $\mathrm{C}_{2} \mathrm{H}_{2}$. The absorption spectrum of $2 \% \mathrm{H}_{2} \mathrm{O}$ and 400 ppm $\mathrm{CO}_{2}$ are also plotted, considering they are the major interfering components for near-infrared atmospheric sensing. Transitions $\mathrm{R}(3)$ at $6046.96 \mathrm{~cm}^{-1}$ and $\mathrm{P}(9)$ at $6534.36 \mathrm{~cm}^{-1}$ are selected as the targets, and they can be easily covered by commercially available laser sources, which are necessary for cost-efficient practical implementation. Influence from atmospheric $\mathrm{H}_{2} \mathrm{O}$ and $\mathrm{CO}_{2}$ on $\mathrm{CH}_{4} / \mathrm{C}_{2} \mathrm{H}_{2}$ sensing would be negligible in cases of the ppm level or higher, such as leakage monitoring.

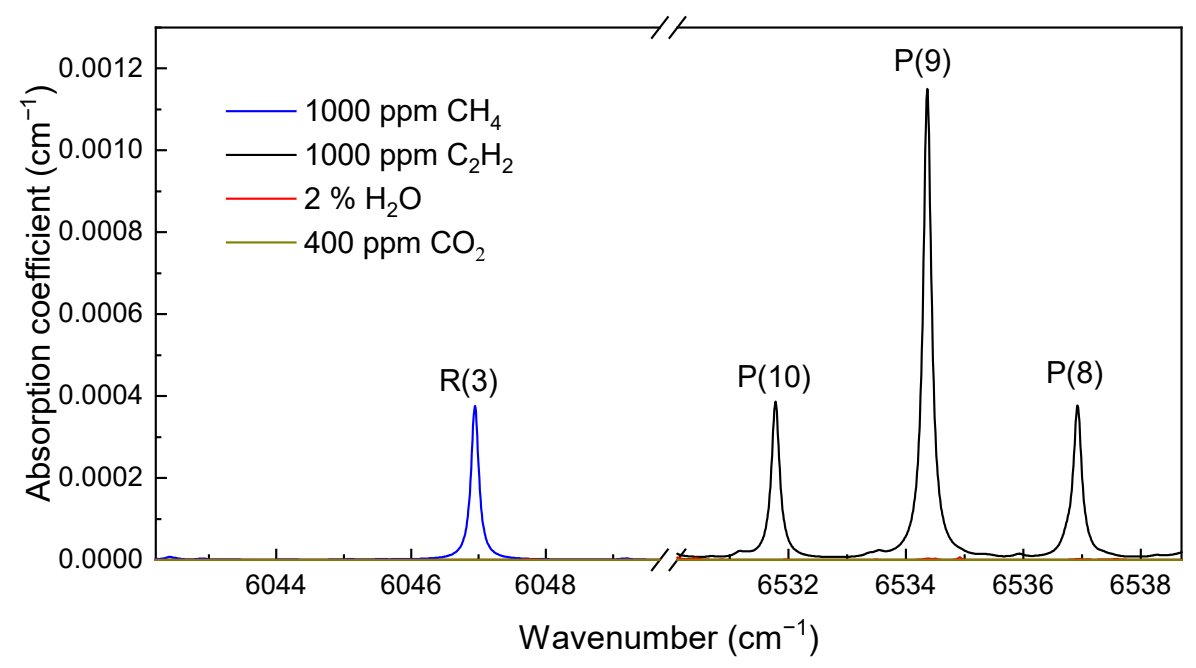

Figure 2. Absorption coefficient simulation for a gas mixture of $1000 \mathrm{ppm} \mathrm{CH}_{4}, 1000 \mathrm{ppm} \mathrm{C}_{2} \mathrm{H}_{2}, 2 \%$ $\mathrm{H}_{2} \mathrm{O}$ and 400 ppm $\mathrm{CO}_{2}$ at $296 \mathrm{~K}$ and $1 \mathrm{~atm}$.

\subsection{Experimental Apparatus}

With the optimal target transitions selected, Figure 3 illustrates the sensing configuration for simultaneous $\mathrm{CH}_{4}$ and $\mathrm{C}_{2} \mathrm{H}_{2}$ leak detection, gas species identification and potential danger warning. The sensing system comprised the monitoring part and a remote probe, connected by a long conductive fiber. Two distributed feedback (DFB) laser diodes with typical emissions at $1653.7 \mathrm{~nm}$ (DFB-LD1) and $1530.4 \mathrm{~nm}$ (DFB-LD2) were controlled by a LabVIEW-based electrical control unit via commercial laser diode drivers (LDC501, Stanford Research Systems). Laser current was modulated by applying a sinusoidal dither to the current ramp at different frequencies for $\mathrm{CH}_{4}$ and $\mathrm{C}_{2} \mathrm{H}_{2}$. The laser beams were combined as one via a $2 \times 150: 50$ fiber coupler and delivered to the work zone via a 3-km optical fiber cable. In the work zone, a custom compact multipass cell was used as a probe, where two quartz mirrors $(\Phi 25.4 \mathrm{~mm})$ coated with dielectric film are $88 \mathrm{~mm}$ apart from each other. The beam was introduced and collected by a pair of $\Phi 1.3 \mathrm{~mm}$ fiber collimators, leading to a 3-m optical path length after 34 reflections in total. Then the harmonic components were generated by laser-sample interaction and were afterwards demodulated by the monitoring part. 


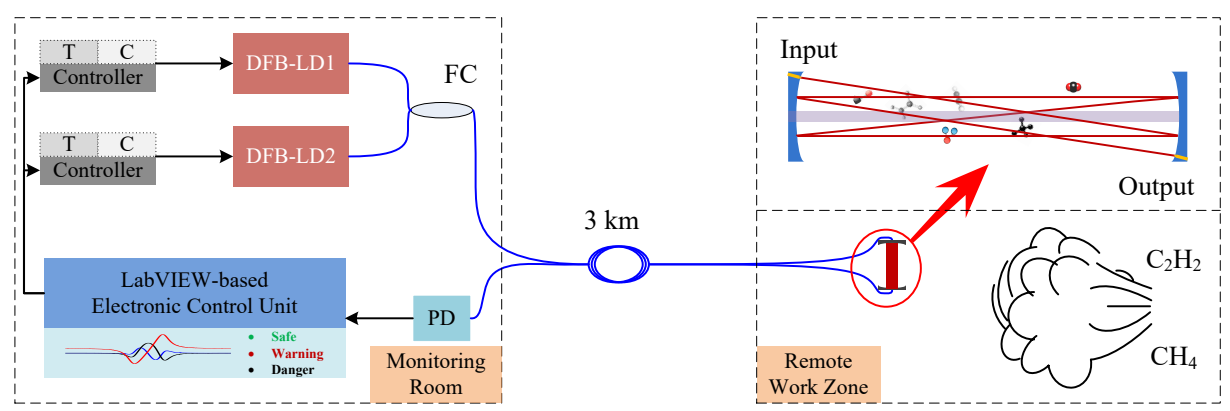

Figure 3. Schematic of the remote sensing system for simultaneous $\mathrm{CH}_{4}$ and $\mathrm{C}_{2} \mathrm{H}_{2}$ detection. T, temperature; C, current; DFB, distributed feedback laser diode; FC, fiber coupler; PD, photodetector.

\subsection{Flow Chart of the Data Retrieval with a LabVIEW-Based Platform}

Data retrievals were performed using the approach described in Section 2, and the processed spectra data and gas concentrations were related by Equation (10), which was then implemented by a LabVIEW-based platform with its flow chart shown in Figure 4. Transmitted laser signal was acquired by a DAQ card after photoelectric conversion. A waveform generator was used to provide sawtooth signals to modulate DFB lasers with independent dither frequencies. The acquired data was then processed by two couples of LIAs for $\mathrm{CH}_{4}$ and $\mathrm{C}_{2} \mathrm{H}_{2}$ respectively.

$$
\left\{\begin{array}{c}
S_{1}=\left.\frac{R_{2 f}}{R_{1 f}}\right|_{\omega=2 \pi f_{1}} \rightarrow \text { Gas }_{1} \\
S_{2}=\left.\frac{R_{2 f}}{R_{1 f}}\right|_{\omega=2 \pi f_{2}} \rightarrow \text { Gas }_{2} \\
S_{n}=\left.\frac{R_{2 f}}{R_{1 f}}\right|_{\omega=2 \pi f_{n}} \cdots
\end{array}\right.
$$

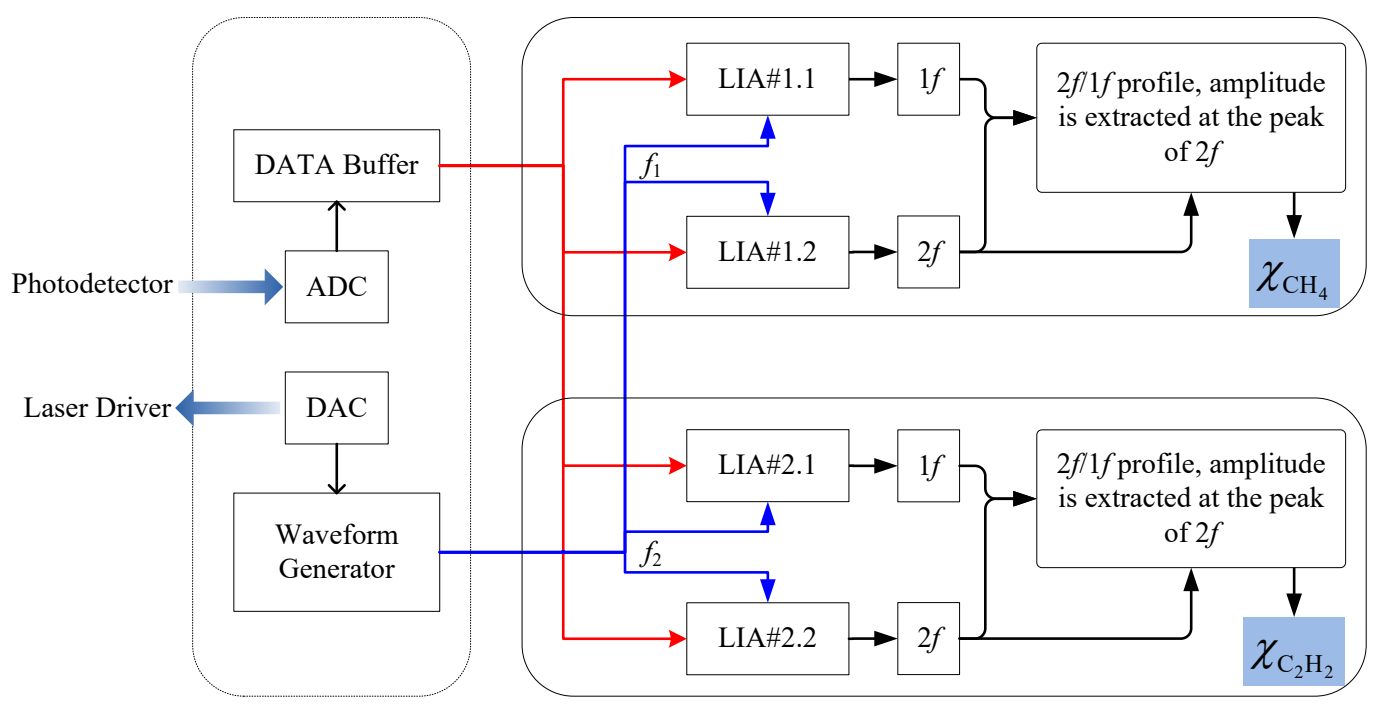

Figure 4. Flow chart of the LabVIEW-based data retrieval. Red lines represent the raw data stream and blue lines represent LIA reference provided by the on-board waveform generator. ADC, analog to digital converter; DAC, digital to analog converter.

LIA\#1.2 was used to extract the second harmonic signal of the raw data at a reference frequency of $f_{1}$, which was generated by the waveform generator. The $2 f / 1 f$ profile was obtained by dividing the magnitude of the first harmonic signal, i.e., output of LIA\#1.1, into the second harmonic magnitude. Note that the $1 f$ and $2 f$ share the same abscissa due to the synchronous trigger. The $2 f / 1 f$ amplitude was extracted at the peak position of 
the $2 f$ profile, corresponding to the absorption transition, for the following $\mathrm{CH}_{4}$ concentration demodulation. Similarly, LIA\#2.1 and LIA\#2.2 were used for $\mathrm{C}_{2} \mathrm{H}_{2}$ concentration demodulation at a reference frequency of $f_{2}$.

\section{Experimental Results}

DFB-LD1 and DFB-LD2 were modulated at $3 \mathrm{kHz}$ and $4 \mathrm{kHz}$ respectively, thus $\mathrm{CH}_{4}$ and $\mathrm{C}_{2} \mathrm{H}_{2}$ measurements could be operated independently without cross-interference. To characterize the sensor, the probe, together with 500-ppm $\mathrm{CH}_{4}$ and 500-ppm $\mathrm{C}_{2} \mathrm{H}_{2}$ diluted in $\mathrm{N}_{2}$ was enclosed inside a gas cell. The $2 f / 1 f$ spectral scans obtained from the detector signal for three different conditions are illustrated in Figure 5. Figure 5a depicts the detected $\mathrm{CH}_{4}$ signals by the first couple of LIAs when both lasers operate and when only the DFB-LD1 operates. The well-matched profiles indicate that simultaneous illumination of both laser beams on the same photodetector does not influence the $\mathrm{CH}_{4}$ detection. For $\mathrm{C}_{2} \mathrm{H}_{2}$ detection, a similar conclusion can also be obtained by the signals measured by the second couple of LIAs, shown in Figure 5b. Therefore, both the $\mathrm{CH}_{4}$ and $\mathrm{C}_{2} \mathrm{H}_{2}$ can be demodulated separately when both lasers operate simultaneously.

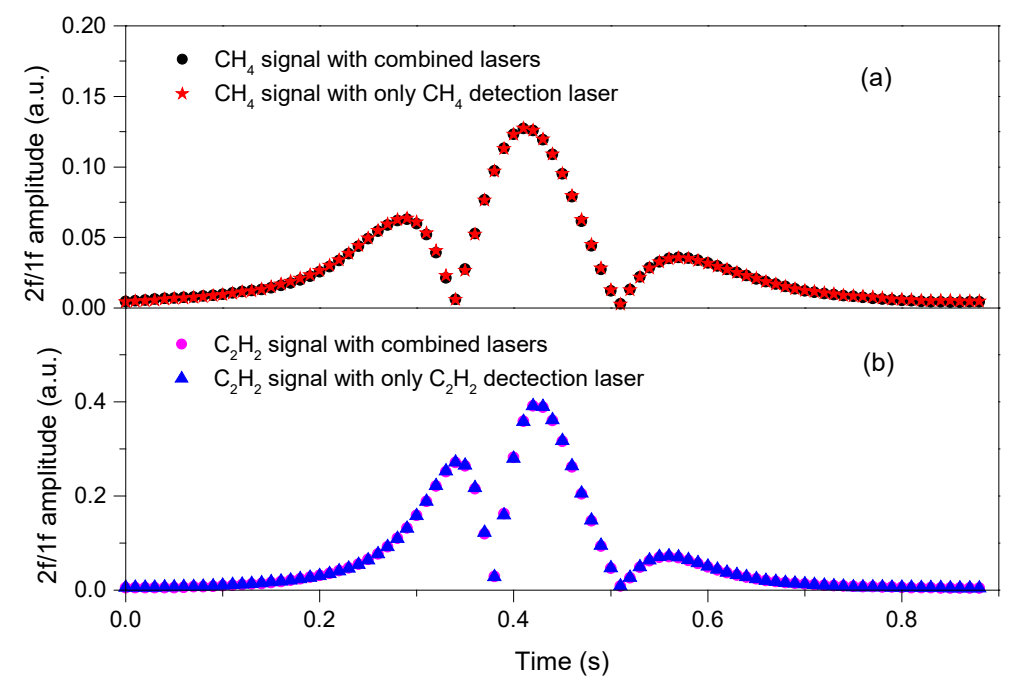

Figure 5. $2 f / 1 f$ spectral profiles for a mixture of (a) 500-ppm $\mathrm{CH}_{4}$ and (b) 500-ppm $\mathrm{C}_{2} \mathrm{H}_{2}$ diluted in $\mathrm{N}_{2}$ at $1 \mathrm{~atm}$ and room temperature, with DFB-LD1 modulated at $3 \mathrm{kHz}$ and DFB-LD2 modulated at $4 \mathrm{kHz}$.

According to Equation (9), the demodulated signal does not relate to the laser intensity. To demonstrate the sensor's immunity to the laser intensity, an experiment was performed with standard sample gases inside the chamber. The laser intensity was attenuated from its maximum down to $90 \%, 70 \%, 50 \%$, and 30\% respectively. Original data were acquired at a sampling rate of $200 \mathrm{kS} / \mathrm{s}$, and the following process was performed according to the flow chart in Figure 4. It took $1 \mathrm{~s}$ for a single measurement, $0.9 \mathrm{~s}$ for data acquisition and $0.1 \mathrm{~s}$ for data processing. The measured $2 f / 1 f$ profiles are illustrated in Figure 6. From the profiles of 500-ppm $\mathrm{CH}_{4}$ shown in Figure 6a, the $2 f / 1 f$ waveforms keep stable with a wide intensity range. The worst correlation coefficient compared to the profile at maximum intensity occurs when the laser intensity is attenuated to $30 \%$, but is still better than 0.9992 . Meanwhile, the correlation coefficients of the $500-p p m \mathrm{C}_{2} \mathrm{H}_{2}$ profiles, shown in Figure $6 \mathrm{~b}$, are calculated to be better than 0.9998 . 

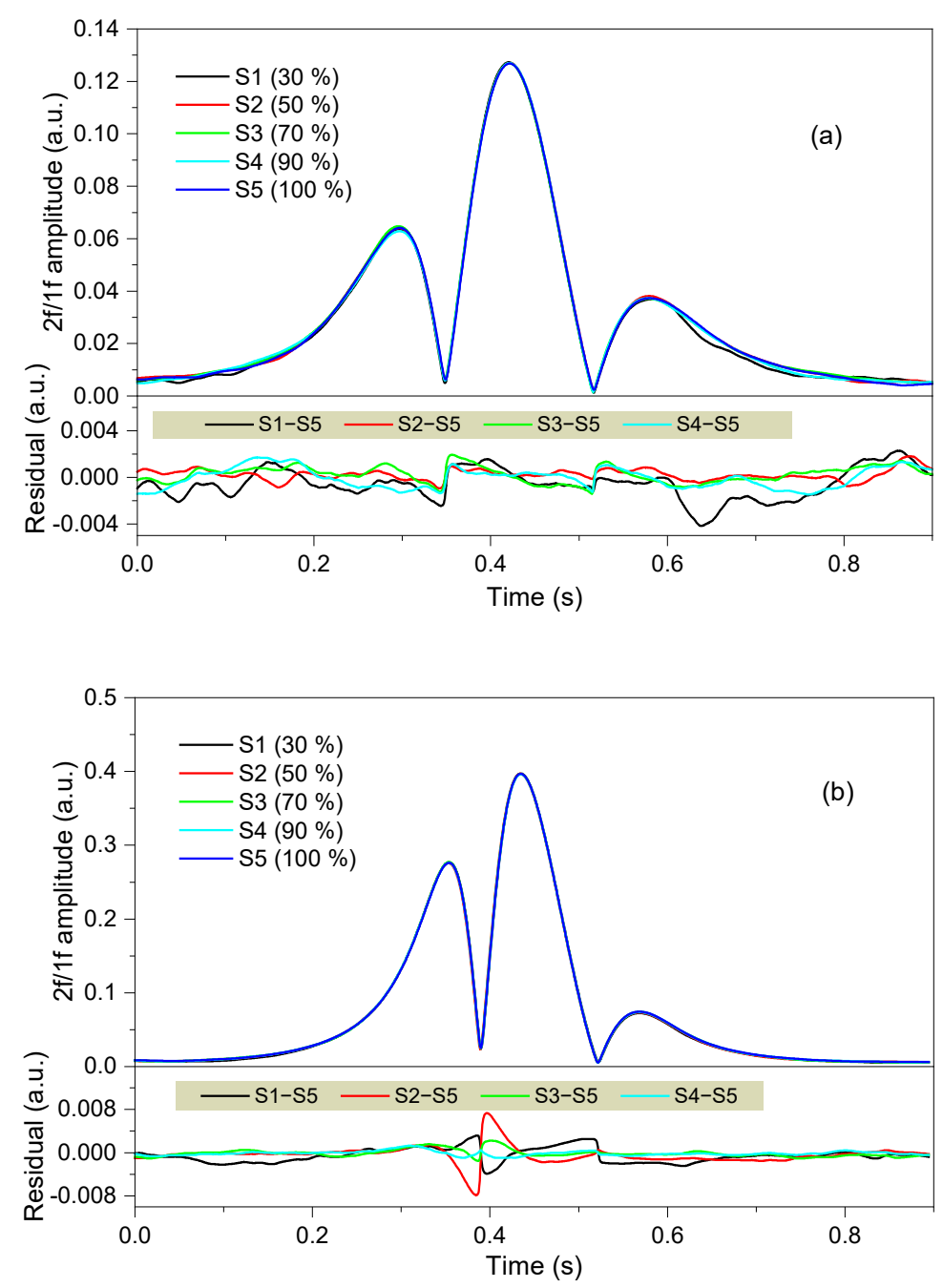

Figure 6. $2 f / 1 f$ spectral profiles with different laser intensities coupled on the same photodetector for (a) 500-ppm $\mathrm{CH}_{4}$ and (b) 500-ppm $\mathrm{C}_{2} \mathrm{H}_{2}$ diluted in $\mathrm{N}_{2}$.

Besides the test with fixed attenuation, its immunity to dynamic laser intensity variation was further investigated by irregularly attenuating the laser beams. With a mixture of 100-ppm $\mathrm{CH}_{4}$ and 100-ppm $\mathrm{C}_{2} \mathrm{H}_{2}$ filled in the chamber, the investigation was performed over $4000 \mathrm{~s}$ with the results shown in Figure 7. The first half of the data with a pale-yellow background was obtained at the condition of drastically intensity variation with an irregular attenuation ranging from 0 to $6 \mathrm{~dB}$, while the second half corresponds to a stable one. From Figure $7 \mathrm{a}$, it can be seen that the measured $\mathrm{CH}_{4}$ data with normalizing process, i.e., $2 f / 1 f$, keep rather stable compared to the conventional $2 f$ signal for gas analysis, and its standard deviation with irregular variation is calculated to be only 1.38 times as much as that with a stable laser intensity. From the $\mathrm{C}_{2} \mathrm{H}_{2}$ data in Figure $7 \mathrm{~b}$, this parameter is calculated to be only 1.23. Therefore, the designed sensor would demonstrate a high immunity to the laser variation coupled on the photodetector in practical application. 


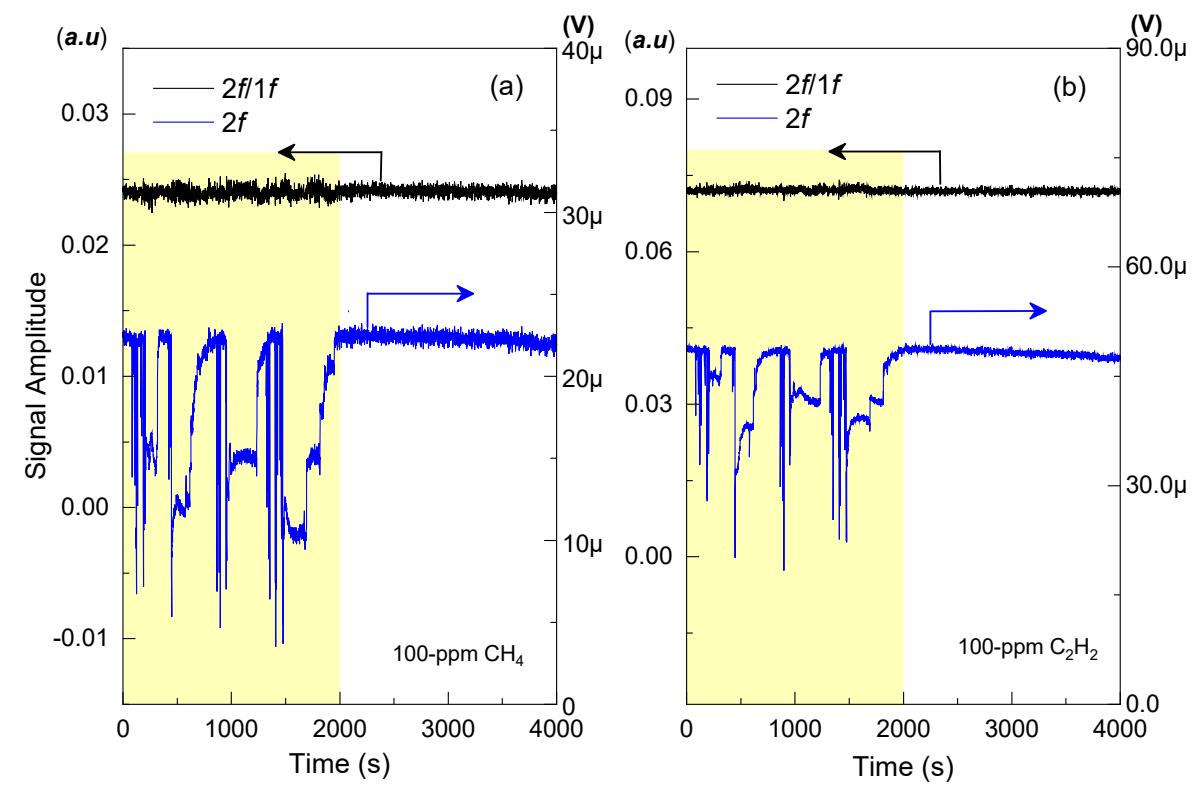

Figure 7. Long-term monitoring of sample gas mixture sealed in the chamber, illustrating a comparison between $2 f / 1 f$ results and $2 f$ results for both (a) 100-ppm $\mathrm{CH}_{4}$ and (b) 100-ppm $\mathrm{C}_{2} \mathrm{H}_{2}$. The left axes pointed by the black arrows mean the amplitude of $2 f / 1 f$ signal, and the right axes pointed by the blue arrows mean the amplitude of $2 f$ signal.

Due to the line-width of an absorption transition, the harmonic signals can be influenced by the modulation depth in its amplitude and spectral waveform. The dependence of $2 f / 1 f$ signal amplitude as a function of the modulation depth was experimentally investigated with the results depicted in Figure 8. The responses for $\mathrm{CH}_{4}$ and $\mathrm{C}_{2} \mathrm{H}_{2}$ measurements are plotted in blue dots and red stars respectively. The $2 f / 1 f$ signal for $\mathrm{CH}_{4}$ increases with modulation depth, but no further increase was observed when the modulation depth is larger than 3.24 GHz. Similarly, the optimum modulation depth for $\mathrm{C}_{2} \mathrm{H}_{2}$ was $5.54 \mathrm{GHz}$. Hence, the modulation depths of $3.24 \mathrm{GHz}$ and $5.54 \mathrm{GHz}$ were selected in the following work to obtain the maximum signals.

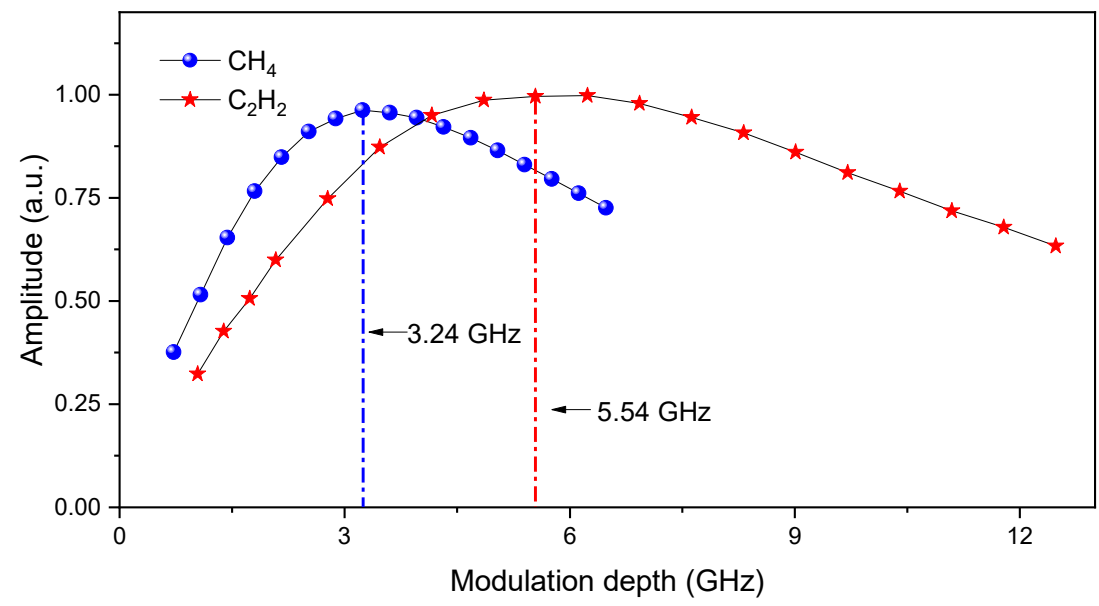

Figure 8. Normalized signal amplitude as a function of modulation depth.

With the optimized modulation depths, the sensor performance was further investigated by measuring a series of $\mathrm{CH}_{4} / \mathrm{C}_{2} \mathrm{H}_{2} / \mathrm{N}_{2}$ mixtures with known concentrations. The mixtures were diluted with $\mathrm{N}_{2}$ using three mass flow meters to different mixing ratios within $1000 \mathrm{ppm}$. The measured signal amplitude as a function of sample gas concentration is depicted in Figure 9a with circle $\left(\mathrm{CH}_{4}\right)$ and square $\left(\mathrm{C}_{2} \mathrm{H}_{2}\right)$ dots. The calculated R-square values are larger than 0.9998 , indicating a good approximation of the linear fitting line 
to the real data points. This implies that the sensor system exhibits an excellent linear response to both $\mathrm{CH}_{4}$ and $\mathrm{C}_{2} \mathrm{H}_{2}$ at atmospheric pressure.
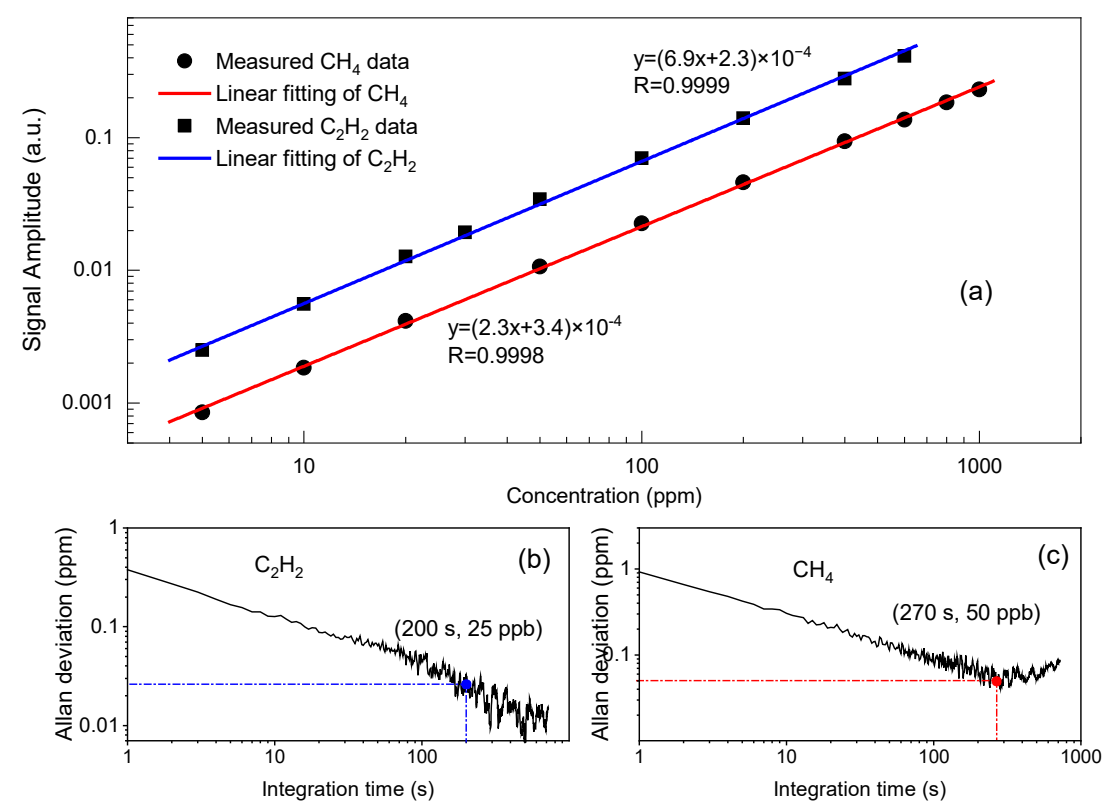

Figure 9. (a) Signal amplitude as a function of sample gas concentration; Allan deviation analysis as a function of integration time for (b) $\mathrm{C}_{2} \mathrm{H}_{2}$ and (c) $\mathrm{CH}_{4}$. The sensor shows a good linear response for both $\mathrm{C}_{2} \mathrm{H}_{2}$ and $\mathrm{CH}_{4}$. The MDLs at $1 \mathrm{~s}$ are $0.4 \mathrm{ppm}$ and $1 \mathrm{ppm}$ for $\mathrm{C}_{2} \mathrm{H}_{2}$ and $\mathrm{CH}_{4}$ respectively. An MDL of $25 \mathrm{ppb}$ for $\mathrm{C}_{2} \mathrm{H}_{2}$ at $200 \mathrm{~s}$ and $50 \mathrm{ppb}$ for $\mathrm{CH}_{4}$ at $270 \mathrm{~s}$ can be obtained.

To evaluate the long-term stability and detection limit of the sensor, an Allan deviation analysis was performed by measuring the signal amplitudes of a gas mixture, comprising 100-ppm $\mathrm{CH}_{4}$ and 20-ppm $\mathrm{C}_{2} \mathrm{H}_{2}$, for one hour. The time resolution of the data acquisition was set to $1 \mathrm{~s}$. Detection limits of the sensor could be further evaluated by the obtained fitting equations in Figure 9a. Figure 9b,c, report the Allan deviation with a minimum detection limit (MDL) of $0.4 \mathrm{ppm}$ for $\mathrm{C}_{2} \mathrm{H}_{2}$ and $1 \mathrm{ppm}$ for $\mathrm{CH}_{4}$ respectively at $1 \mathrm{~s}$ integration time. Furthermore, an MDL of $25 \mathrm{ppb}$ for $\mathrm{C}_{2} \mathrm{H}_{2}$ at $200 \mathrm{~s}$ and $50 \mathrm{ppb}$ for $\mathrm{CH}_{4}$ at $270 \mathrm{~s}$ respectively was reported, exhibiting good stability for the current system.

After characterization and calibration with the above experiments, the open-ended probe was installed in a work zone to verify its capability of monitoring hydrocarbon leakage. With a $3 \mathrm{~km}$ optical fiber cable, the other parts, except the sensing probe, were operated in a monitoring room at $23 \pm 1{ }^{\circ} \mathrm{C}$ temperature and $50 \pm 10 \% \mathrm{RH}$, which is a moderate operation condition for the DFB lasers and most other electronic equipment. The hydrocarbon leaked from two gas cylinders, $1 \% \mathrm{CH}_{4}$ and $0.1 \% \mathrm{C}_{2} \mathrm{H}_{2}$, were both diluted in $\mathrm{N}_{2}$. The maximum concentrations are much lower than the explosion limits, i.e., $5 \%$ for $\mathrm{CH}_{4}$ and $2.3 \%$ for $\mathrm{C}_{2} \mathrm{H}_{2}$, for security. By employing two mass flow meters, the $\mathrm{CH}_{4}$ leakage was actively controlled at $0.75 \mathrm{~L} / \mathrm{min}$ from $t_{1}, 1.5 \mathrm{~L} / \mathrm{min}$ from $t_{3}$, then was closed at $t_{5}$. For $\mathrm{C}_{2} \mathrm{H}_{2}$, the leakage was $0.5 \mathrm{~L} / \mathrm{min}$ from $t_{2}$ and rose to $1 \mathrm{~L} / \mathrm{min}$ from $t_{4}$, then was closed at $t_{6}$. Figure 10 illustrates the recorded time history curves with different leakage times, different leakage rates, and different gas species. We observed the $\mathrm{CH}_{4}$ at around $200 \mathrm{ppm}$ after $\mathrm{t}_{1}$, then an apparent increase to about $300 \mathrm{ppm}$ after $t_{3}$, finally a decline from $t_{5}$. Similarly, the $\mathrm{C}_{2} \mathrm{H}_{2}$ was observed at about $10 \mathrm{ppm}$ after $\mathrm{t}_{2}$, an ascent to about $20 \mathrm{ppm}$ after $\mathrm{t}_{4}$, then a gradual decline from $t_{6}$. The curves' tendency matches the leakage conditions well, indicating its high practicability in real-time remote multi-species monitoring applications. 


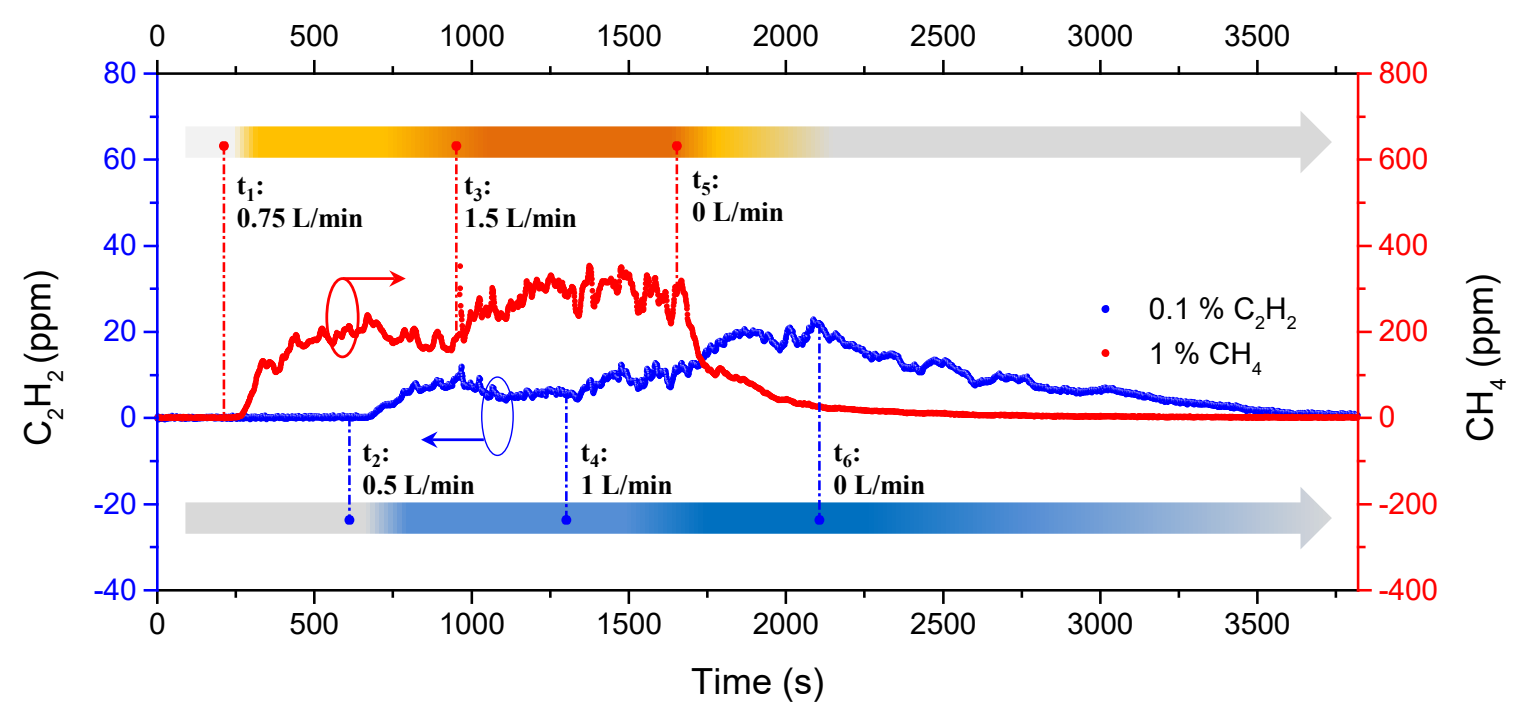

Figure 10. Time history curves of recorded $\mathrm{CH}_{4}$ and $\mathrm{C}_{2} \mathrm{H}_{2}$ concentration at different leakage conditions. $t_{1}$ to $\mathrm{t}_{6}$ represent different leakage rates at different times, and the leakage rate can be controlled by two mass flow meters. The arrows in yellow and blue illustrate the measured relative leakage over time and the dark color means more leakage than light color.

\section{Conclusions}

In summary, we developed a practical spectroscopic sensor combining normalized wavelength modulation spectroscopy and frequency-division multiplexing for real-time multi-species monitoring. The first harmonic signal was employed to suppress the intensity noise in order to improve the immunity to irregular laser intensity noise. Different gas species in the sensing area can be remotely demodulated at separate frequencies without any cross-interference. A centimeter-size multi-pass cell with a 3-m optical length was developed as a compact terminal probe. $\mathrm{CH}_{4}$ and $\mathrm{C}_{2} \mathrm{H}_{2}$ were chosen to demonstrate the sensor's characterization and performance. Two DFB laser diodes, emitting at $1653.7 \mathrm{~nm}$ and $1530.4 \mathrm{~nm}$, were independently modulated at $3 \mathrm{kHz}$ and $4 \mathrm{kHz}$ to target the $\mathrm{R}(3)$ transition of $\mathrm{CH}_{4}$ and $\mathrm{P}(9)$ transition of $\mathrm{C}_{2} \mathrm{H}_{2}$ respectively. After optimizing the wavelength modulation depth, the combined laser beam can simultaneously interrogate the $\mathrm{CH}_{4}$ and $\mathrm{C}_{2} \mathrm{H}_{2}$ with sub-ppm MDLs with $1 \mathrm{~s}$ time resolution. Furthermore, an application test for remote hydrocarbon leakage monitoring was carried out with a good match between the recorded results and the controllable leakage conditions, indicating promising and reliable remote multi-species sensing in real-world applications. Besides, since the sensing system operates with silica-based single-mode fiber in near-infrared, covering absorption transitions of many different gas species [28], the current sensor can be easily extended to monitor more industrial hazardous gas species without scarifying the sensing performance.

Author Contributions: Conceptualization, W.J.; methodology, W.J. and Q.W.; software, Q.W.; validation, W.J. and H.Z.; formal analysis, W.J. and J.L.; investigation, M.H. (Mai Hu) and M.H. (Mengpeng $\mathrm{Hu}$ ); mechanical structure, M.H. (Mai Hu) and Y.W.; data curation, W.J.; writing-original draft preparation, W.J.; writing-review and editing, R.K. and Q.W.; supervision, R.K. and Q.W.; project administration, M.H. (Mai Hu) and Q.W.; funding acquisition, J.L. and Q.W. All authors have read and agreed to the published version of the manuscript.

Funding: This research was funded by the Strategic Priority Research Program of Chinese Academy of Sciences (Grant no. XDA17040513), the Scientific Instrument Developing Project of the Chinese Academy of Sciences (Grant no. YJKYYQ20190037), the National Key Research and Development Project (Grant no. 2019YFB2006003), Research on the Highly Sensitive Laser On-line Monitoring System of Key Atmospheric Component Isotopes in the Qinghai-Tibet Plateau (Grant no. 2019QZKK020802) and the National Natural Science Foundation of China (Grant no. 62005267).

Institutional Review Board Statement: Not applicable. 
Informed Consent Statement: Not applicable.

Data Availability Statement: The data that support the plots within this paper are available from the corresponding author on request basis.

Conflicts of Interest: The authors declare no conflict of interest.

\section{References}

1. Wang, B.; Wu, C.; Reniers, G.; Huang, L.; Kang, L.; Zhang, L. The future of hazardous chemical safety in China: Opportunities, problems, challenges and tasks. Sci. Total Environ. 2018, 643, 1-11. [CrossRef] [PubMed]

2. Wan, J.; Yu, Y.; Wu, Y.; Feng, R.; Yu, N. Hierarchical leak detection and localization method in natural gas pipeline monitoring sensor networks. Sensors 2012, 12, 189-214. [CrossRef]

3. Wang, Z.; Wang, Q.; Ching, J.Y.L.; Wu, J.C.Y.; Zhang, G.; Ren, W. A portable low-power QEPAS-based $\mathrm{CO}_{2}$ isotope sensor using a fiber-coupled interband cascade Lasers. Sensor. Actuators B. Chem. 2017, 246, 710-715. [CrossRef]

4. Huang, Y.; Sinha, A.; Zhao, H.; Dang, X.; Zhang, Y.; Quan, X. Real time detection of hazardous hydroxyl radical using an electrochemical approach. Chem. Select. 2019, 4, 12507-12511. [CrossRef]

5. Ma, X.; Zou, J.; Li, W.; He, J.J. Miniature spectrometer based on a Fourier transform spectrometer chip and a commercial photodetector array. Chin. Opt. Lett. 2019, 17, 123001. [CrossRef]

6. Werle, P.; Slemr, F.; Maurer, K.; Kormann, R.; Mücke, R.; Jänker, B. Near-and mid-infrared laser-optical sensors for gas analysis. Opt. Lasers Eng. 2002, 37, 101-114. [CrossRef]

7. Hodgkinson, J.; Tatam, R.P. Optical gas sensing: A review. Meas. Sci. Technol. 2013, 24, 012004. [CrossRef]

8. Wu, H.; Yin, X.; Dong, L.; Pei, K.; Sampaolo, A.; Patimisco, P.; Zheng, H.; Ma, W.; Zhang, L.; Yin, W. Simultaneous dual-gas QEPAS detection based on a fundamental and overtone combined vibration of quartz tuning fork. Appl. Phys. Lett. 2017, 110, 121104. [CrossRef]

9. Liu, K.; Mei, J.; Zhang, W.; Chen, W.; Gao, X. Multi-resonator photoacoustic spectroscopy. Sensor. Actuators B. Chem. 2017, 251, 632-636. [CrossRef]

10. Rieker, G.B.; Giorgetta, F.R.; Swann, W.C.; Kofler, J.; Zolot, A.M.; Sinclair, L.C.; Baumann, E.; Cromer, C.; Petron, G.; Sweeney, C.; et al. Frequency-comb-based remote sensing of greenhouse gases over kilometer air paths. Optica 2014, 1, 290. [CrossRef]

11. Wang, J.; Sun, C.; Wang, G.; Zou, M.; Tan, T.; Liu, K.; Chen, W.; Gao, X. A fibered near-infrared laser heterodyne radiometer for simultaneous remote sensing of atmospheric $\mathrm{CO}_{2}$ and $\mathrm{CH}_{4}$. Opt. Lasers Eng. 2020, 129, 106083. [CrossRef]

12. Tommasi, E.D.; Casa, G.; Gianfrani, L. An Intensity-Stabilized Diode-Laser Spectrometer for Sensitive Detection of $\mathrm{NH}_{3}$. IEEE Trans. Instrum. Meas. 2007, 56, 309-312. [CrossRef]

13. Zhu, C.; Chang, J.; Wang, P.; Wei, W.; Wang, Q.; Wang, F.; Zhang, S. Continuously wavelength-tunable light source with constant-power output for elimination of residual amplitude modulation. IEEE Sens. J. 2015, 15, 316-321. [CrossRef]

14. Wang, Q.; Chang, J.; Kong, D.L.; Liu, Y.N.; Wang, F.P.; Zhu, C.G.; Wei, W.; Liu, X.Z. Optical Measurement of Water Vapor Concentration and Gas Pressure. IEEE Sens. J. 2014, 14, 563-569. [CrossRef]

15. Chang, H.; Chang, J.; Huang, Q.; Wang, Q.; Tian, C.; Wei, W.; Liu, Y. Immunity to laser power variation in a DFB diode laser based optical gas sensor using a division process. Sensors 2015, 15, 9582-9591. [CrossRef]

16. Rieker, G.B.; Jeffries, J.B.; Hanson, R.K. Calibration-free wavelength-modulation spectroscopy for measurements of gas temperature and concentration in harsh environments. Appl. Opt. 2009, 48, 5546-5560. [CrossRef] [PubMed]

17. Sun, K.; Chao, X.; Sur, R.; Goldenstein, C.; Jeffries, J.; Hanson, R. Analysis of calibration-free wavelength-scanned wavelength modulation spectroscopy for practical gas sensing using tunable diode lasers. Meas. Sci. Technol. 2013, 24, 125203. [CrossRef]

18. Spearrin, R.M.; Ren, W.; Jeffries, J.B.; Hanson, R.K. Multi-band infrared $\mathrm{CO}_{2}$ absorption sensor for sensitive temperature and species measurements in high-temperature gases. Appl. Phys. B Lasers 2014, 116, 855-865. [CrossRef]

19. Li, H.; Rieker, G.B.; Liu, X.; Jeffries, J.B.; Hanson, R.K. Extension of wavelength-modulation spectroscopy to large modulation depth for diode laser absorption measurements in high-pressure gases. Appl. Opt. 2006, 45, 1052-1061. [CrossRef] [PubMed]

20. Liu, Y.; Chang, J.; Lian, J.; Liu, Z.; Wang, Q.; Zhu, C. A time difference method for measurement of phase shift between distributed feedback laser diode (DFB-LD) output wavelength and intensity. Sensors 2015, 15, 16153-16161. [CrossRef]

21. Zhu, C.; Wang, G.; Zheng, Z.; Wang, R.; Tao, X.; Wang, P. A Method for Real-Time Monitoring of Inherent System Loss Designed for FLRDS-Based Gas Sensors. IEEE Photonics J. 2016, 8, 1-8. [CrossRef]

22. Friedlein, J.T.; Baumann, E.; Briggman, K.A.; Colacion, G.M.; Giorgetta, F.R.; Goldfain, A.M.; Herman, D.I.; Hoenig, E.V.; Hwang, J.; Newbury, N.R. Dual-comb photoacoustic spectroscopy. Nat. Commun. 2020, 11, 1-10. [CrossRef] [PubMed]

23. Coddington, I.; Newbury, N.; Swann, W. Dual-comb spectroscopy. Optica 2016, 3, 414-426. [CrossRef]

24. Cossel, K.C.; Waxman, E.M.; Giorgetta, F.R.; Cermak, M.; Coddington, I.R.; Hesselius, D.; Ruben, S.; Swann, W.C.; Truong, G.W.; Rieker, G.B. Open-path dual-comb spectroscopy to an airborne retroreflector. Optica 2017, 4, 724-728. [CrossRef]

25. Chen, K.; Guo, M.; Liu, S.; Zhang, B.; Deng, H.; Zheng, Y.; Chen, Y.; Luo, C.; Tao, L.; Lou, M. Fiber-optic photoacoustic sensor for remote monitoring of gas micro-leakage. Opt. Express 2019, 27, 4648-4659. [CrossRef]

26. Tombez, L.; Zhang, E.; Orcutt, J.; Kamlapurkar, S.; Green, W. Methane absorption spectroscopy on a silicon photonic chip. Optica 2017, 4, 1322-1325. [CrossRef] 
27. Schmidt, F.M.; Vaittinen, O.; Metsälä, M.; Kraus, P.; Halonen, L. Direct detection of acetylene in air by continuous wave cavity ring-down spectroscopy. Appl. Phys. B Lasers 2010, 101, 671-682. [CrossRef]

28. Gordon, I.E.; Rothman, L.S.; Hill, C.; Kochanov, R.V.; Tan, Y.; Bernath, P.F.; Birk, M.; Boudon, V.; Campargue, A.; Chance, K.V.; et al. The HITRAN2016 molecular spectroscopic database. J. Quant. Spectrosc. RA 2017, 203, 3-69. [CrossRef] 